nature immunology

\title{
Neuroimmune communication
}

\begin{abstract}
We present a special set of Review articles on neuroimmune communication that highlight how the immune system and nervous system are anatomically connected, mechanistically communicate and reciprocally influence the other's function.
\end{abstract}

o mmunology and neuroscience have historically been separate fields of study, each with its own particular lingo, with few researchers readily conversant in both fields. Immune responses generated in the central nervous system (CNS) are distinct from those that arise in the periphery. Specific anatomical barriers and tissue-resident glial cells protect the brain parenchyma from potentially harmful immune-cellmediated damage. Yet, some means of immunological control exists within the CNS that endows the ability to confront and contain various neurotropic pathogens or, when dysregulated, contributes to neuroinflammatory disease states. Recent studies have shown that the peripheral immune system and the nervous system can communicate using common molecular signaling cues. This month, Nature Immunology and Nature Neuroscience present a joint online focus (http://www.nature. com/focus/neuroimmune_communication) composed of a series of commissioned Review articles that examine the communication between the immune system and nervous system.

Veiga-Fernandes and Pachnis introduce the concept of the enteric neuroimmune cell unit that forms a vast 'sensory organ' and serves to protect and maintain intestinal integrity and function. The enteric neuroimmune system provides both innate defense and memory responses to specific pathogens. Similar extracellular cues instruct the development of enteric neuroprogenitor and hematopoietic cells to form this system during gestation. Further maturation of this system occurs in the post-natal period when the gut is colonized by commensal microbes, which elicit reciprocal signaling between neuronal-glial cells and tissueresident immune cells.

Hsiao and colleagues continue that theme by describing how the gut microbiome influences the function of peripheral immune cells and CNS-resident cells, as well as its effect on brain development and disease pathogenesis. Commensal microbes produce metabolites, including short-chain fatty acids and aryl hydrocarbon ligands, which can act distally within the CNS on glial cells, affecting both their development and their function. Reciprocally, neuroendocrine mediators released via the hypothalamic-pituitary-adrenal axis influence intestinal permeability, immune-cell activation and microbiome composition. There is also evidence of microbial dysbiosis in neurological and psychiatric disorders which might contribute in part to disease, although additional research is needed to discern the underlying mechanisms and to what extent the microbiota has a causal role.

Engelhardt and colleagues describe immunological privilege displayed by the CNS toward peripheral immune cells during homeostatic conditions. They draw distinctions between the lymphatic drainage of cerebrospinal fluid, which bathes the meninges and cerebral ventricles of the brain, versus the lack of such lymphatic drainage of interstitial fluid that bathes parenchymal CNS tissues. The authors provide a detailed anatomical description of the cellular and acellular brain barriers that restrict cellular access to the CNS parenchyma in human and rodent brains, as well as a discussion of afferent and efferent trafficking of cells and solutes within the perivascular areas of these barrier sites.

Prinz and Priller discuss how peripheral immune cells can enter the CNS under more pathological scenarios. Blood-borne immune cells are typically not found in the healthy CNS; instead, tissue-resident microglia, meningeal and perivascular macrophage populations provide immunosurveillance and can produce innate immunological mediators. These innate immune responses are common characteristics of neurodegenerative disorders. However, autoimmune disease, infection or injury alters the permeability of the blood-brain barrier, which enables the entry of activated adaptive immune cells into the CNS via fenestrated blood capillaries, where they contribute to disease progression.

Klein and colleagues explore neurological impairments that can arise after acute infection by neurotropic pathogens has been resolved. Acute infection leads to the release of inflammatory cytokines by astrocytes and microglia and by infiltrating leukocytes that are recruited to the sites of infection. Many inflammatory mediators can affect the integrity of the blood-brain barrier and induce symptoms such as fatigue, hypersomnia and inability to concentrate. Although antimicrobial treatment can eradicate the pathogens, chronic inflammation can persist unabated within the CNS and produce behavioral changes, including impaired learning and memory, depression and other mood disorders. Such findings prompt questions about whether various neurological diseases are mediated in part by an immunological component.

Whereas immunological mediators can influence neuronal function, Pavlov and Tracey discuss mechanisms whereby neural pathways regulate peripheral immunity. Many types of immune cells express receptors for neurotransmitters released by neurons, such as acetylcholine or catecholamines. Such mediators can elicit anti-inflammatory responses by suppressing the production of pro-inflammatory cytokines via 'inflammatory reflex' pathways. Neural circuits can likewise promote systemic immunosuppression after injury or stroke, affect lymphocyte trafficking and enhance tissue protection by altering macrophage function. Studies have also revealed how the brain integrates information on immunological and metabolic status. In turn, the brain exerts top-down controlfor example, via the reward circuitry - on activation of the peripheral immune system, which can promote protective responses to infection.

This Focus only presents a small fraction of what is already known, and much remains unknown. This an exciting area of research that demands a truly interdisciplinary approach aimed at elucidating the bidirectional cellular and molecular interactions between these two systems in the context of development and disease. 